\title{
Ruin probabilities in a finite-horizon risk model with investment and reinsurance
}

\author{
R. Romera and W. Runggaldier* \\ University Carlos III de Madrid and University of Padova
}

July 3, 2012

\begin{abstract}
A finite horizon insurance model is studied where the risk/reserve process can be controlled by reinsurance and investment in the financial market. Our setting is innovative in the sense that we describe in a unified way the timing of the events, that is the arrivals of claims and the changes of the prices in the financial market, by means of a continuous-time Semi-Markov process (SMP) which appears to be more realistic than, say, classical diffusion-based models. Obtaining explicit optimal solutions for the minimizing ruin probability is a difficult task. Therefore we derive a specific methodology, based on recursive relations for the ruin probability, to obtain a reinsurance and investment policy that minimizes an exponential bound (Lundbergtype bound) on the ruin probability.
\end{abstract}

Keywords: Risk process, Semi-Markov processes, optimal reinsurance and investment, Lundberg-type bounds.

MSC: 91B30, 93E20, 60J28.

*Partially supported by the Spanish ME grant SEJ2007-64500. Part of the contribution by this author was obtained while he was visiting professor 2009 for the chair Quantitative Finance and Insurance at the LMU University in Munich funded by LMU Excellent. Hospitality and financial support are gratefully acknowledged. 


\section{Introduction}

We consider a discrete-time insurance risk/reserve process which can be controlled by reinsurance and investment in the financial market, and we study the ruin probability problem in the finite horizon case. Although controlling a risk/reserve process is a very active area of research (see Chen et al. (2000), Wang et al. (2004), Schmidli (2008), Huang et al. (2009) and references therein), obtaining explicit optimal solutions minimizing the ruin probability is in general a difficult task even for the classical Cramér-Lundberg risk process. Thus, an alternative method commonly used in ruin theory is to derive inequalities for ruin probabilities. The inequalities can be used to obtain upper bounds for the ruin probabilities (see Willmot \& Lin (2001), Grandell (1991), Schmidli (2002)), and this is the approach followed in the present paper. The basis of this approach is the well known fact that in the classical Cramér-Lundberg model, if the claim sizes have finite exponential moments, then the ruin probability decays exponentially as the initial surplus increases (see for instance the book by Asmussen (2000)). For the heavy-tailed claims' case it is also shown to decay with a rate depending on the distribution of the claim size; see e.g., Gaier et al. (2003). Paulsen (1998) reviews general processes for the ruin problem when the insurance company invests in a risky asset. Xiong \& Yang (2011) give conditions for the ruin probability to be equal to 1 for any initial endowment and without any assumption on the distribution of the claim size as long as it is not identically zero.

Control problems for risk/reserve processes are commonly formulated in continuous time. Schäl (2004) introduces a formulation of the problem where events (arrivals of claims and asset price changes) occur at discrete points in time that may be deterministic or random, but their total number is fixed. Diasparra \& Romera (2009) consider a similar formulation in discrete time. Having a fixed total number of events implies that in the case of random time points the horizon is random as well. In the present paper we follow an approach inspired by Edoli \& Runggaldier (2010) who claim that a more natural way to formulate the problem in case of random time points is to consider a given fixed time horizon so that also the number of event times becomes random and this makes the problem nonstandard. Accordingly it is reasonable to assume that also the control decisions (level of reinsurance and amount invested) correspond to these random time points. Notice that this formulation can be seen equivalently in discrete or continuous time.

The stochastic elements that affect the evolution of the risk/reserve pro- 
cess are thus the timing and size of the claims as well as the dynamics of the prices of the assets in which the insurer is investing. This evolution is controlled by the sequential choice of the reinsurance and investment levels.

Claims occur at random points in time and also their sizes are random, while asset price evolutions are usually modeled as continuous time processes. On small time scales, prices actually change at discrete random time points and vary by tick size. In the proposed model we let also asset prices change only at discrete random time points with their sizes being random as well. This will allow us to consider the timing of the events, namely the arrivals of claims and the changes of the asset prices, to be triggered by a same continuous-time semi-Markov process, i.e. a stochastic process where the embedded jump chain (the discrete process registering what values the process takes) is a Markov Chain, and where the holding times (time between jumps) are random variables, whose distribution function may depend on the two states between which the move is made. Since between event times the situation for the insurer does not change, we shall consider controls only at event times.

Our bounds are derived mainly for the purpose of obtaining a reinsurance and investment policy that possibly minimizes the ruin probability. These bounds may not be particularly useful as bounds for the ruin probability itself since they are not guaranteed to be less than 1 , they make it however possible to actually derive a reinsurance and investment policy that, by minimizing the bounds, may be considered as a reasonable policy in view of minimizing the ruin probability. This is confirmed by the simulation results in Piscitello (2012), where one can also see that, in line with the recent literature, investing in the financial market may be dangerous. The main computational advantage of minimizing the bounds is that, while the actual optimal policy may turn out to be of the form of a closed-loop feedback policy and thus difficult to determine computationally, the bound minimizing policy is of the myopic type requiring to minimize locally the bound at each event time and it is thus of a much simpler structure.

The rest of the paper is organized as follows. In section 2 we describe the model and, in particular, the risk process. Section 3 is devoted to derive recursive relations for the ruin probability. On the basis of these recursive relations, in section 4 we obtain exponential (Lundberg-type) bounds on the ruin probability. In section 5 we then discuss an approach of the type of policy iteration in order to determine the insurance and investment levels that minimize these bounds. Finally, section 6 contains some concluding 
remarks.

\section{The model}

We consider a finite time horizon $T>0$. More precisely, to model the timing of the events (arrival of claims and asset price changes), inspired by Schäl (2005) we introduce the process $\left\{K_{t}\right\}_{t>0}$ for $t \leq T$, a continuous time semiMarkov process (SMP) on $\{0,1\}$, where $K_{t}=0$ holds for the arrival of a claim, and $K_{t}=1$ for a change in the asset price. The embedded Markov chain, i.e., the jump chain associated to the SMP $\left\{K_{t}\right\}_{t>0}$, evolves according to a transition probability matrix $P=\left\|p_{i j}\right\|_{i, j \in\{0,1\}}$ that is supposed to be given, and the holding times (time between jumps) are random variables whose probability distribution function may depend on the two states between which the move is made. We come back to this point in the next subsection 2.1.

Let $T_{n}$ be the random time of the $n-t h$ event, $n \geq 1$, and let the counting process $N_{t}$ denote the number of events having occurred up to time $t$, defined as follows

$$
N_{t}=\sum_{j=1}^{\infty} 1_{\left\{T_{j} \leq t\right\}}
$$

and so

$$
T_{n}=\min \left\{t \geq 0 \mid N_{t}=n\right\}
$$

\section{$2.1 \quad$ Risk process}

In this section we introduce the dynamics of the controlled risk process $X_{t}$ for $t \in[0, T]$ with $T$ a given fixed horizon. For this purpose let $Y_{n}$ be the $n-t h(n \geq 1)$ claim payment represented by a sequence of independent and identically distributed (i.i.d.) random variables with common probability distribution function (p.d.f.) $F(y)$ having support in the positive half line. Let $Z_{n}$ be the random variable denoting the time between the occurrence of the $n-1$ st and $n$th $(n \geq 1)$ jumps of the SMP $\left\{K_{t}\right\}_{t>0}$. We assume that $\left\{Z_{n}\right\}$ is a sequence of i.i.d. random variables with p.d.f. $G(\cdot)$. From this we may consider that the transition probabilities of the SMP $\left\{K_{t}\right\}_{t>0}$ are

$$
P\left\{K_{T_{n+1}}=j, Z_{n+1} \leq s \mid K_{T_{n}}=i\right\}=p_{i j} G(s)
$$


Notice that for a full SMP model, the distribution function $G(\cdot)$ depends also on $i$ and $j$ and the results derived below go through in the same way also for this more general case. Since in many cases of interest (see Example 1 below) $G(\cdot)$ is independent of $i$ and $j$, for simplicity of presentation we shall restrict ourselves to such a situation.

Example 1 A specific form of SMP, which we shall refer to also later, arises for example as follows: let $N_{t}^{0}$ and $N_{t}^{1}$ be independent Poisson processes with intensities $\lambda_{0}$ and $\lambda_{1}$ respectively. We may think of $N_{t}^{0}$ as counting the number of claims and $N_{t}^{1}$ that of price changes and we have that $N_{t}=N_{t}^{0}+N_{t}^{1}$ is again a Poisson process with intensity $\lambda=\lambda_{0}+\lambda_{1}$. We may then put

$$
K_{T_{n}}= \begin{cases}0 & \text { if at } T_{n} \text { a jump of } N_{t}^{0} \text { has occurred (claim) } \\ 1 & \text { if at } T_{n} \text { a jump of } N_{t}^{1} \text { has occurred (price change) }\end{cases}
$$

It then follows easily that

$$
\begin{aligned}
& P\left\{K_{T_{n+1}}=j, Z_{n+1} \leq s \mid K_{T_{n}}=i\right\} \\
& =P\left\{K_{T_{n+1}}=j \mid Z_{n+1} \leq s, K_{T_{n}}=i\right\} P\left\{Z_{n+1} \leq s \mid K_{T_{n}}=i\right\} \\
& =p_{i j} P\left\{Z_{1} \leq s\right\}=\frac{\lambda_{j}}{\lambda}\left[1-e^{-\lambda s}\right]
\end{aligned}
$$

so that, in this case

$$
\left\{\begin{array}{l}
p_{i j}=\frac{\lambda_{j}}{\lambda}:=p_{j}, \quad \forall i \\
G(s)=\left[1-e^{-\lambda s}\right]
\end{array}\right.
$$

Remark 2 Our model, in particular the specific case of Example 1, does not allow for simultaneous jumps (claims and price changes may however occur very close to one another). On the other hand we could include simultaneous jumps into our model by extending $K_{t}$ to take three possible values, namely

$$
K_{T_{n}}= \begin{cases}0 & \text { for a claim } \\ 1 & \text { for a price change } \\ 2 & \text { for simultaneous claim and price change }\end{cases}
$$


The risk process is controlled by reinsurance and investment. In general this means that we may choose adaptively at the event times $T_{N_{t}}$ (they correspond to the jump times of $N_{t}$ ) the retention level (or proportionality factor or risk exposure) $b_{N_{t}}$ of a reinsurance contract as well as the amount $\delta_{N_{t}}$ to be invested in the risky asset, namely in $S_{N_{t}}$ with $S_{t}$ denoting discounted prices. For the values $b$ that the various $b_{N_{t}}$ may take we assume that $b \in\left[b_{\min }, 1\right] \subset(0,1]$, where $b_{\text {min }}$ will be introduced below and for the values of $\delta$ for the various $\delta_{N_{t}}$ we assume $\delta \in[\underline{\delta}, \bar{\delta}]$ with $\underline{\delta} \leq 0$ and $\bar{\delta}>0$ exogenously given. Notice that this condition allows also for negative values of $\delta$ meaning that, see also Schäl (2004), short selling of stocks is allowed. On the other hand, with an exogenously given upper bound $\bar{\delta}$, it might occasionally happen that $\delta_{N_{t}}>X_{N_{t}}$ implying a temporary debt of the agent beyond his/her current wealth in order to invest optimally in the financial market. By choosing a policy that minimizes the ruin probability, this debt is however only instantaneous and with high probability leads to a positive wealth already at the next event time.

Assume that prices change only according to

$$
\frac{S_{N_{t}+1}-S_{N_{t}}}{S_{N_{t}}}=\left(e^{W_{N_{t}+1}}-1\right) K_{T_{N_{t}+1}}
$$

where $W_{n}$ is a sequence of i.i.d. random variables taking values in $[\underline{w}, \bar{w}]$ with $-\infty<\underline{w}<0<\bar{w}<+\infty$ and with p.d.f. $H(w)$. For simplicity and without loss of generality we consider only one asset to invest in. An immediate generalization would be to allow for investment also in the money market account.

Let $c$ be the premium rate (income) paid by the customer to the company,f fixed in the contract. Since the insurer pays to the reinsurer a premium rate, which depends on the retention level $b_{N_{t}}$ chosen at the various event times $T_{N_{t}}$, we denote by $C\left(b_{N_{t}}\right)$ the net income rate of the insurer at time $t \in[0, T]$. For $b \in\left[b_{\min }, 1\right]$ we let $h(b, Y)$ represent the part of the generic claim $Y$ paid by the insurer and in what follows we take the function $h(b, Y)$ to be of the form $h(b, Y)=b \cdot Y$ (proportional reinsurance). We shall call policy a sequence $\pi=\left(b_{n}, \delta_{n}\right)$ of control actions, where $\left(b_{n}, \delta_{n}\right)$ is short for $\left(b_{T_{n}}, \delta_{T_{n}}\right)$. Control actions over a single period will be denoted by $\phi_{n}=$ $\left(b_{n}, \delta_{n}\right)$. According to the expected value principle with safety loading $\theta$ of the reinsurer, for a given starting time $t<T$, the income rate function $C(b)$ 
can be chosen as follows:

$$
C(b):=c-(1+\theta) \frac{E\left\{Y_{1}-h\left(b, Y_{1}\right)\right\}}{E\left\{Z_{1} \wedge(T-t)\right\}}, 0<t<T
$$

Note that $C(b)$ depends on the starting and the terminal times $t$ and $T$ only via $T-t$ in the denominator of (2.4); the value of $T-t$ can however be considered as given so that $C(b)$ can indeed be considered as depending only on the current value $b$ of the retention level (it is in fact a rate over the interval $[t, T])$.

We use $Z_{1}$ and $Y_{1}$ in the above formula since, by our i.i.d. assumption, the various $Z_{n}$ and $Y_{n}$ are all independent copies of $Z_{1}$ and $Y_{1}$. Notice also that, in order to keep formula (2.4) simple and possibly similar to standard usage, in the denominator of the right hand side we have considered the random time $Z_{1}$ between to successive events, while more correctly we should have taken the random time between two successive claims, which is larger. For this we can however play with the safety loading factor. In fact, if we denote by $\bar{Z}$ the average time between successive claims before $T$ and, for a given $\theta$ put $\bar{\theta}=(1+\theta) \frac{\bar{Z}}{E\left\{Z_{1} \wedge(T-t)\right\}}-1$ we have that $\frac{(1+\theta)}{E\left\{Z_{1} \wedge(T-t)\right\}}=\frac{(1+\bar{\theta})}{\bar{Z}}$. Since in this way $1+\bar{\theta}=(1+\theta) \frac{\bar{Z}}{E\left\{Z_{1} \wedge(T-t)\right\}}$ and $\bar{Z}>E\left\{Z_{1} \wedge(T-t)\right\}$, we are assured that $(1+\bar{\theta})>1$. We can now define

$$
b_{\min }:=\min \left\{b \in\left[b^{*}, 1\right] \mid c \geq C(b) \geq c^{*}\right\},
$$

where $c^{*} \geqslant 0$ denotes the minimal value of the premium considered by the insurer and $b^{*}>0$ is the minimal value for the proportion of the claim paid by the insurer. It follows that $C\left(b_{\min }\right)=\max \left[c^{*}, C\left(b^{*}\right)\right]$. We make the

\section{Assumption 3 Let}

i) The tuple $\left(Z_{n}, Y_{n}, W_{n}\right)_{n \geq 1}$ is formed by independent sequences of i.i.d. random variables.

ii) Defining $\bar{r}:=\sup \left\{r \geq 0 \mid E\left\{e^{r Y_{1}}\right\}<+\infty\right\}$ assume that $\bar{r}>0$ and that $E\left\{e^{\bar{r} Y_{1}}\right\}=+\infty$.

iii) $c-(1+\theta) \frac{E\left\{Y_{1}\right\}}{E\left\{Z_{1} \wedge T\right\}}>0$.

Remark 4 Notice that 
i) Since $b \leq 1$, point ii) in Assumption 3 implies that for all $b \in\left[b_{\text {min }}, 1\right]$ one has $E\left\{e^{r b Y_{1}}\right\}<+\infty$ for $r \in[0, \bar{r})$ and $E\left\{e^{r b Y_{1}}\right\}=+\infty$ for $r=\frac{\bar{r}}{b_{\min }}$.

ii) Since the support of $Y_{1}$ is in the positive half line, we have $\lim _{r \uparrow \bar{r}} E\left\{e^{r Y_{1}}\right\}=$ $+\infty$. Notice that $\bar{r}$ may be equal to $+\infty$, e.g. if the support of $Y_{1}$ is bounded.

iii) For $h(b, Y)=b Y$, point iii) in Assumption 3 implies that $c \geqslant C(b) \geqslant$ $c^{*} \geqslant 0, \forall b \in\left[b_{\text {min }}, 1\right]$ and that, furthermore, $c \geqslant 0$.

iv) From the definition of $C(b)$ in (2.4), the definition of $b_{\min }$ in (2.5) and by point iii) in Assumption 3, in addition to $C\left(b_{\min }\right)=\max \left[c^{*}, C\left(b^{*}\right)\right]$, it follows that

$$
b_{\min }=\left\{\begin{array}{lll}
1-\left(c-c^{*}\right) \frac{E\left\{Z_{1} \wedge(T-t)\right\}}{(1+\theta) E\left\{Y_{1}\right\}} & \text { if } \quad c^{*}>C\left(b^{*}\right)>0 \\
b^{*} & \text { if } \quad 0<c^{*} \leq C\left(b^{*}\right)
\end{array}\right.
$$

In the given setting we obtain now for the insurance risk process (surplus) $X$ the following one-step transition dynamics between the generic random times $T_{n}$ and $T_{n+1}$ when at $T_{n}$ a control action $\phi=(b, \delta)$ is taken for a certain $b \in\left[b_{\min }, 1\right] \subset(0,1]$, and $\delta \in[\underline{\delta}, \bar{\delta}]$,

$$
X_{T_{n+1}}=X_{T_{n}}+C(b) Z_{n+1}-\left(1-K_{T_{n+1}}\right) h\left(b, Y_{n+1}\right)+K_{T_{n+1}} \delta\left(e^{W_{n+1}}-1\right)
$$

Definition 5 Letting $U:=\left[b_{\min }, 1\right] \times[\underline{\delta}, \bar{\delta}]$, we shall say that a control action $\phi=(b, \delta)$ is admissible if $(b, \delta) \in U$. Notice that $U$ is compact. A policy $\pi$ will be called admissible if it implies admissible control actions.

We want now to express the one-step dynamics in (2.7) when starting from a generic time instant $t<T$ with a capital $x$. For this purpose note that if, for a given $t<T$ one has $N_{t}=n$, the time $T_{N_{t}}$ is the random time of the $n-t h$ event and $T_{n} \leq t \leq T_{n+1}$. Since, when standing at time $t$, we observe the time that has elapsed since the last event in $T_{N_{t}}$, it is not restrictive to assume that $t=T_{N_{t}}$ (see the comment below after (2.8)). A further justification for letting $t=T_{N_{t}}$ can be given in the case of Example 1: the random variable there has a negative exponential distribution and this distribution is memoryless. Furthermore, since $Z_{n}, Y_{n}, W_{n}$ are i.i.d., in the 
one-step random dynamics for the risk process $X_{t}$ we may replace the generic $\left(Z_{n+1}, Y_{n+1}, W_{n+1}\right)$ by $\left(Z_{1}, Y_{1}, W_{1}\right)$. We may thus write

$$
X_{N_{t}+1}=x+C(b) Z_{1}-\left(1-K_{T_{N_{t}+1}}\right) h\left(b, Y_{1}\right)+K_{T_{N_{t}+1}} \delta\left(e^{W_{1}}-1\right)
$$

for $0<t<T, T>0$ and with $X_{t}=x \geq 0$ (recall that we assumed $t=T_{N_{t}}$ ). Notice that, if we had $t \neq T_{N_{t}}$ and therefore $t>T_{N_{t}}$, the second term on the right in 2.8 would become $C(b)\left[Z_{1}-\left(t-T_{N_{t}}\right)\right]$ and 2.8 could then be rewritten as

$X_{N_{t}+1}=\left[x-C(b)\left(t-T_{N_{t}}\right)\right]+C(b) Z_{1}-\left(1-K_{T_{N_{t}+1}}\right) h\left(b, Y_{1}\right)+K_{T_{N_{t}+1}} \delta\left(e^{W_{1}}-1\right)$

with the quantity $\left[x-C(b)\left(t-T_{N_{t}}\right)\right]$, which is known at time $t$, replacing $x$. This is the sense in which above we mentioned that it is not restrictive to assume that $t=T_{N_{t}}$. In what follows we shall work with the risk process $X_{t}$, (or $\left.X_{N_{t}}\right)$ as defined by (2.8). For convenience we shall denote by $\left(b_{n}, \delta_{n}\right)$ the values of $\phi=(b, \delta)$ at $t=T_{N_{t}}$. Accordingly, we shall also write $\left(b_{N_{t}}, \delta_{N_{t}}\right)$ for $\left(b_{T_{N_{t}}}, \delta_{T_{N_{t}}}\right)$.

Objective: Determine an admissible reinsurance and investment policy $\pi$ so as to minimize a suitable bound on the ruin probability, where by "ruin" we mean the event when the wealth process first becomes zero.

Following Schmidli (2008) we shall also introduce an absorbing (cemetery) state that we take as the state $x=0$; in fact, once the company has been ruined, it is reasonable to assume that its wealth remains at the level zero.

\section{Recursions}

We start this section by specifying some notation and introducing the basic definitions concerning our ruin probabilities.

\subsection{Notation and Definitions}

In view of our ultimate goal of obtaining bounds on the ruin probability over a given finite time horizon $[t, T]$, we first introduce the notion of probability of ruin before $T$ within the first $n$ events. Recalling that ruin occurs as soon as $X_{t}=0$ and that $X_{t}$ remains at level zero after ruin, we give the following more formal 
Definition 6 Assume we are standing at time $t<T$ with a surplus value of $X_{t}=X_{T_{N_{t}}}=x>0$ and with $K_{t}=K_{T_{N_{t}}}=k \in\{0,1\}$. Given an admissible reinsurance and investment policy $\pi$, we shall denote by $\psi_{n}^{\pi}(t, x ; k)$ the probability of ruin before $T$ within the first $n$ events and let it be given by

$$
\psi_{n}^{\pi}(t, x, k)=P_{t, x, k}^{\pi}\left\{X_{n} \leq 0 ; T_{n} \leq T\right\}
$$

where $P_{t, x ; k}^{\pi}$ denotes the probability conditional on $X_{t}=x>0, K_{t}=k \in$ $\{0,1\}$ and for a given admissible policy $\pi$.

Our first purpose in the next section is to obtain a recursive relation for $\psi_{n}^{\pi}(t, x ; k)$.

\subsection{Recursive relations}

In formula (3.1) of Definition 6 it was implicit that $t<T$. When in what follows we shall write $\psi_{n-1}^{\pi}\left(T_{1}, X_{1} ; K_{1}\right)$, we shall mean the random variable, function of $\left(T_{1}, X_{1} ; K_{1}\right)$, given by

$$
\psi_{n-1}^{\pi}\left(T_{1}, X_{1} ; K_{1}\right)=P_{T_{1}, X_{1}, K_{1}}^{\pi}\left\{X_{n} \leq 0 ; T_{n} \leq T\right\}
$$

on the event $T_{1} \leq T$.

We now have the following recursion result

Proposition 7 For an initial surplus $x$ at a given time $t \in[0, T]$, as well as an initial event $K_{T_{N_{t}}}=k$ and a given admissible policy $\pi$, for any $n \geq 1$ we have

$$
\psi_{n}^{\pi}(t, x, k) \leq \psi_{1}^{\pi}(t, x, k)+P\left\{T_{1} \leq T\right\} E_{t, x, k}^{\pi}\left\{\psi_{n-1}^{\pi}\left(T_{1}, X_{1}, K_{1}\right)\right\}
$$

Proof: We have the decomposition

$$
\begin{aligned}
\psi_{n}^{\pi}(t, x, k)= & P_{t, x, k}^{\pi}\left\{X_{n} \leq 0, T_{n} \leq T\right\} \\
= & P_{t, x, k}^{\pi}\left\{X_{n} \leq 0, T_{n} \leq T, X_{1} \leq 0, T_{1} \leq T\right\} \\
& \quad+P_{t, x, k}^{\pi}\left\{X_{n} \leq 0, T_{n} \leq T, X_{1}>0, T_{1} \leq T\right\}
\end{aligned}
$$


For the first term on the right we have

$$
\begin{aligned}
& P_{t, x, k}^{\pi}\left\{X_{n} \leq 0, T_{n} \leq T, X_{1} \leq 0, T_{1} \leq T\right\} \\
& =P_{t, x, k}^{\pi}\left\{T_{n} \leq T \mid X_{1} \leq 0, T_{1} \leq T\right\} \cdot P_{t, x, k}^{\pi}\left\{X_{1} \leq 0, T_{1} \leq T\right\} \\
& =P\left\{T_{n} \leq T \mid T_{1} \leq T\right\} \psi_{1}^{\pi}(t, x, k)=\frac{P\left\{T_{n} \leq T\right\}}{P\left\{T_{1} \leq T\right\}} \psi_{1}^{\pi}(t, x, k) \\
& =\frac{P\left\{N_{T}-N_{T_{1}} \geq n-1\right\}}{P\left\{T_{1} \leq T\right\}} \psi_{1}^{\pi}(t, x, k) \\
& =\frac{P\left\{N_{T}-N_{t} \geq n\right\}}{P\left\{T_{1} \leq T\right\}} \psi_{1}^{\pi}(t, x, k) \leq \psi_{1}^{\pi}(t, x, k)
\end{aligned}
$$

where we have used the fact that

$$
P\left\{N_{T}-N_{t} \geq n\right\} \leq P\left\{N_{T}-N_{t} \geq 1\right\}=P\left\{Z_{1} \leq T-t\right\}=P\left\{T_{1} \leq t\right\}
$$

and that the probability of events related to $T_{n}$ (equivalently to $N_{t}$ ) does not depend neither on $\pi$ nor on $(t, x, k)$.

Taking into account (3.2) and that it holds on the event $T_{1} \leq T$, for the second term we have instead (by $\mathcal{F}_{1}^{T, X, K}$ we denote the $\sigma$-algebra generated by $\left(T_{m}, X_{m}, K_{m}\right)$ up to $\left.t=T_{1}\right)$

$$
\begin{aligned}
& P_{t, x, k}^{\pi}\left\{X_{n} \leq 0, T_{n} \leq T, X_{1}>0, T_{1} \leq T\right\} \leq P_{t, x, k}^{\pi}\left\{X_{n} \leq 0, T_{n} \leq T, T_{1} \leq T\right\} \\
& =E_{t, x, k}^{\pi}\left\{E^{\pi}\left\{\mathbf{1}_{\left\{X_{n} \leq 0, T_{n} \leq T\right\}} \mathbf{1}_{\left\{T_{1} \leq T\right\}} \mid \mathcal{F}_{1}^{T, X, K}\right\}\right\} \\
& =E_{t, x, k}^{\pi}\left\{\mathbf{1}_{\left\{T_{1} \leq T\right\}} E^{\pi}\left\{\mathbf{1}_{\left\{X_{n} \leq 0, T_{n} \leq T\right\}} \mid \mathcal{F}_{1}^{T, X, K}\right\}\right\} \\
& =E_{t, x, k}^{\pi}\left\{\mathbf{1}_{\left\{T_{1} \leq T\right\}} P_{T_{1}, X_{1}, K_{1}}^{\pi}\left\{X_{n} \leq 0, T_{n} \leq T\right\}\right\} \\
& =P\left\{T_{1} \leq T\right\} E_{t, x, k}^{\pi}\left\{\psi_{n-1}^{\pi}\left(T_{1}, X_{1}, K_{1}\right)\right\}
\end{aligned}
$$

where we have also used the fact that $T_{1}$ is independent of the other random variables. 


\section{Bounds}

We derive here bounds on the ruin probability in a general setting and, in the next section 5, we then minimize them with respect to the reinsurance and investment policy. We base ourselves on results in Diasparra \& Romera (2009) and Diasparra \& Romera (2010) that are here extended to the general setup of the present paper. To stress the fact that the process $X$ defined in 2.7 corresponds to the choice of a specific policy $\pi$, in what follows we shall use the notation $X^{\pi}$.

Given an admissible policy $\pi_{t}=\left(b_{t}, \delta_{t}\right)$ and defining for $t \in[0, T]$ the random variable

$$
V_{t}^{\pi}:=C(b)\left(Z_{1} \wedge(T-t)\right)-\mathbf{1}_{\left\{Z_{1} \leq T-t\right\}}\left[\left(1-K_{T_{N_{t}}+1}\right) b Y_{1}-K_{T_{N_{t}}+1} \delta\left(e^{W_{1}}-1\right)\right]
$$

where $b=b_{t}$ and $\delta=\delta_{t}$ let, for $r \in\left[0, \frac{\bar{r}}{b_{\min }}\right)$ and $k \in\{0,1\}$

$$
l_{r}^{\pi}(t, k):=E_{t, x, k}\left\{e^{-r V_{t}^{\pi}}\right\}-1
$$

which does not depend on $x$ and where, for reasons that should become clear below, we distinguish the dependence of $l^{\pi}$ on $r$ from that on $(t, k)$.

Remark 8 Notice that (see Remark 4, i) and ii)) $\lim _{r \uparrow \frac{\bar{r}}{b_{\min }}} l_{r}^{\pi}(t, k)=+\infty$ for all $(t, k)$.

Definition 9 We shall call a policy $\pi$ strongly admissible and denote their set by $\mathcal{A}$ if at each $t \in[0, T]$ the corresponding control action $\left(b_{t}, \delta_{t}\right) \in U$ and, for any $(t, k) \in[0, T] \times\{0,1\}$, it holds that $E_{t, k}^{\pi}\left\{V_{t}^{\pi}\right\}>0$.

Notice that $\mathcal{A}$ is non-empty since, see Assumption 3 , iii), it contains at least the stationary policy $\left(b_{N_{t}}, \delta_{N_{t}}\right) \equiv\left(b_{\min }, 0\right)$.

Proposition 10 For each $(t, k) \in[0, T] \times\{0,1\}$ and each $\pi \in \mathcal{A}$ we have that

i) As a function of $r \in\left[0, \frac{\bar{r}}{b_{\min }}\right)$ with $\bar{r}$ such that Assumption 3, ii) is satisfied, $l_{r}^{\pi}(t, k)$ is convex with a negative slope at $r=0$;

ii) the equation $l_{r}^{\pi}(t, k)=0$, seen as an equation in $r$, has a unique positive root in $\left(0, \frac{\bar{r}}{b_{\min }}\right)$ that we denote by $R^{\pi}(t, k)$, so that the defining relation for $R^{\pi}(t, k)$ is

$$
l_{R^{\pi}(t, k)}^{\pi}(t, k)=0 \quad \forall t \in[0, T], k \in\{0,1\}
$$


Proof: Differentiating with respect to $r$ under the expectation sign leads to

$$
\left\{\begin{array}{l}
\frac{\partial}{\partial r}\left(l_{r}^{\pi}(t, k)\right)_{\mid r=0}=E_{t, x, k}\left\{-V_{t}^{\pi}\right\}<0 \\
\frac{\partial^{2}}{\partial r^{2}}\left(l_{r}^{\pi}(t, k)\right)=E_{t, x, k}\left\{\left(V_{t}^{\pi}\right)^{2} \cdot e^{-r V_{t}^{\pi}}\right\}>0
\end{array}\right.
$$

where the first inequality follows from the admissibility of $\pi$ (see Definition 91), and so statement i) follows immediately. In view of ii) notice that from Assumption 3, ii) (see also Remark 4, i) and ii)) one obtains $\lim _{r \uparrow \frac{\bar{r}}{b_{\min }}} l_{r}^{\pi}(t, k)=+\infty$. This fact, combined with i) leads to ii).

Definition 11 For given $\pi \in \mathcal{A}$ and $t \in[0, T]$ let

$$
R_{t}^{\pi}:=\inf _{t \leq s \leq T} \min \left[R^{\pi}(s, 0), R^{\pi}(s, 1)\right]
$$

In relation to this definition see also Remark 20,

Remark 12 Notice that by Remark 8 we may always assume that $R^{\pi}(t, k)<$ $\frac{\bar{r}}{b_{\min }}, \forall(t, k) \in[0, T] \times\{0,1\}$ and thus also $R_{t}^{\pi}<\frac{\bar{r}}{b_{\min }}$.

We derive now our first bound in the following

Lemma 13 Given an initial time $t \in[0, T]$ and an initial event $k \in\{0,1\}$, we have

$$
\psi_{1}^{\pi}(t, x, k) \leq e^{-R_{t}^{\pi} x}, \quad \forall x>0, \pi \in \mathcal{A}
$$

where $R_{t}^{\pi}$ is as in Definition 11 (see (4.5)).

Proof: Notice first that, from (2.7) and its definition in (4.1), the random variable $V_{t}^{\pi}$ represents the increment of the risk process $X_{t}$ between two successive event times, provided these event times occur within the interval $[t, T]$. According to Definition 6 we then obtain

$$
\begin{aligned}
\psi_{1}^{\pi}(t, x, k) & =P_{t, x, k}^{\pi}\left\{X_{1}<0, T_{1} \leq T\right\} \leq P_{t, x, k}^{\pi}\left\{X_{1}<0\right\} \\
& =P_{t, x, k}^{\pi}\left\{V_{t}^{\pi} \leq-x\right\}
\end{aligned}
$$

On the other hand, by Chebyshev's inequality we have for all $r>0$

$$
P_{t, x, k}^{\pi}\left\{V_{t}^{\pi} \leq-x\right\}=P_{t, x, k}^{\pi}\left\{e^{-r V_{t}^{\pi}} \geq e^{r x}\right\} \leq e^{-r x} E_{x, k}^{\pi}\left\{e^{-r V_{t}^{\pi}}\right\}
$$


For $r=R^{\pi}(t, k)$, using the previous two relations, one obtains

$$
\psi_{1}^{\pi}(t, x, k) \leq e^{-R^{\pi}(t, k) x} E_{t, x, k}^{\pi}\left\{e^{-R^{\pi}(t, k) V_{t}^{\pi}}\right\} \leq e^{-R^{\pi}(t, k) x} \leq e^{-R_{t}^{\pi} x}
$$

where in the last relation we have used Definition 11 and Proposition 10 (ii).

Next put

$$
\gamma:=P\left\{N_{T}-N_{t} \geq 1\right\}=P\left\{Z_{1} \leq T-t\right\}=P\left\{T_{1} \leq T\right\}
$$

and notice that this $\gamma$ is independent of $(\pi, x, k)$ and it holds that $\gamma<1$. We now have the further

Lemma 14 Given an initial surplus $x>0$ at a given time $t \in[0, T]$, we have, for all $n \in \mathbb{N}$ and any initial event $k \in\{0,1\}$ and for all $\pi \in \mathcal{A}$,

$$
\psi_{n}^{\pi}(t, x, k) \leq\left(\sum_{m=0}^{n-1} \gamma^{m}\right) e^{-R_{t}^{\pi} x}
$$

Proof: The proof is by induction. By Lemma 13 the statement is true for $n=1$. Assume it is true for $n-1$, namely $\psi_{n-1}^{\pi}(t, x, k) \leq\left(\sum_{m=0}^{n-2} \gamma^{m}\right) e^{-R_{t}^{\pi} x}$ for any $x>0, k \in\{0,1\}$ and $\pi \in \mathcal{A}$. Given the Definition 11 for $R_{t}^{\pi}$, we may formulate the induction hypothesis also as follows

$$
\psi_{n-1}^{\pi}\left(T-s, X_{1}, K_{1}\right) \leq\left(\sum_{m=0}^{n-2} \gamma^{m}\right) e^{-R_{t}^{\pi} X_{1}}, \quad \forall s \in[0, T-t]
$$

By Proposition 7 as well as the Lemma 13 , and taking into account the definition of $R_{t}^{\pi}$ in (4.5) and that of $V_{t}^{\pi}$ in (4.1), we then obtain

$$
\begin{aligned}
& \psi_{n}^{\pi}(t, x, k) \leq e^{-R_{t}^{\pi} x}+P\left\{T_{1} \leq T\right\}\left(\sum_{m=0}^{n-2} \gamma^{m}\right) E_{t, x, k}^{\pi}\left\{e^{-R_{t}^{\pi} X_{1}}\right\} \\
& \leq e^{-R_{t}^{\pi} x}+\gamma\left(\sum_{m=0}^{n-2} \gamma^{m}\right) e^{-R_{t}^{\pi} x} E_{t, x, k}^{\pi}\left\{e^{-R_{t}^{\pi} V_{t}^{\pi}}\right\} \leq\left(\sum_{m=0}^{n-1} \gamma^{m}\right) e^{-R_{t}^{\pi} x}
\end{aligned}
$$

where the last inequality follows from the definitions of $R^{\pi}(t, k)$ and $R_{t}^{\pi}$ as well as the properties of the function $l_{r}^{\pi}(t, k)$ stated in Proposition 10, In fact, from $l_{R^{\pi}(t, k)}^{\pi}(t, k)=0$ (see (4.3)) and $R_{t}^{\pi} \leq R^{\pi}(t, k)$ it follows that $l_{R_{t}^{\pi}}^{\pi}(t, k)=E_{t, x, k}^{\pi}\left\{e^{-R_{t}^{\pi} V_{t}^{\pi}}\right\}-1 \leq 0$.

As an immediate consequence of Lemma 14 we obtain now our main 
Theorem 15 Given an initial surplus $x>0$ at a given time $t \in[0, T]$, we have, for all $n \in \mathbb{N}$ and any initial event $k \in\{0,1\}$ and for all $\pi \in \mathcal{A}$

$$
\psi_{n}^{\pi}(t, x, k) \leq \frac{1}{1-\gamma} e^{-R_{t}^{\pi} x}
$$

Remark 16 Notice that the bound in Theorem 15 will in many cases be larger than 1 and thus not useful as a numerical bound on the amount of the probability of ruin itself. The main purpose for deriving this bound is however to obtain a reinsurance and investment policy that keeps the ruin probability low and in the next section 5 we derive it as the policy that minimizes the bound. With this goal in mind there is no need to derive the tightest possible bound; what is important however is that the coefficient in the right hand side of (4.9) is independent of $(\pi, x, k)$. Notice also that, while the true optimal policy is most likely of the form of a feedback policy and thus difficult to determine, the policy derived from minimizing the bound is of the form of a myopic policy, which is much easier to derive as we shall show in the next section.

Remark 17 Notice that, see Remark 12, for $t \rightarrow T$ the right hand side in 4.9) is bounded from below by $e^{-\frac{\bar{r}}{b_{\min }} x}$ which is strictly positive unless $\bar{r}=$ $+\infty$ (the latter happens whenever the support of $Y_{1}$ is bounded, see Remark 4. ii)). On the other hand, always for $t \rightarrow T$, we have $\psi_{n}^{\pi}(t, x, k) \rightarrow 0$. This hints at the possibility of viewing the bound in Theorem 15 also as having the flavor of an infinite time ruin probability in the spirit of the Lundberg bound, although we work on a finite time horizon.

\section{Optimizing the bounds}

As mentioned previously, it is in general a difficult task to obtain an explicit solution to the reinsurance-investment problem in order to minimize the ruin probability even for the classical risk process. We shall thus choose the reinsurance and investment levels in order to minimize the bounds that we have derived. By Theorem 15 this amounts to choosing a strategy $\pi \in \mathcal{A}$ such that for each $t \in[0, T]$ the value of $R_{t}^{\pi}$ is as large as possible. In order to achieve this goal notice that, by Proposition 10 the function $l_{r}^{\pi}(t, k)$ is, as a function of $r \in\left[0, \frac{\bar{r}}{b_{\min }}\right)$ (for every fixed $(t, k) \in[0, T] \times\{0,1\}$ and $\pi \in \mathcal{A}$ ), convex with a zero in $r=0$ and (see Remark 12) a unique positive one in 
$R^{\pi}(t, k) \in\left[0, \frac{\bar{r}}{b_{\min }}\right)$. To obtain, for a given $t \in[0, T]$, the largest value of $R_{t}^{\pi}=\inf _{t \leq s \leq T} \min \left[R^{\pi}(s, 0), R^{\pi}(s, 1)\right]$ it thus suffices to choose $\pi \in \mathcal{A}$ that minimizes $l_{r}^{\pi}(t, k)$ at $r=R_{t}^{\pi}$. This appeals also to intuition in the sense that, by its definition in 4.2 , minimizing $l_{r}^{\pi}(t, k)$ amounts to penalizing negative values of $V_{t}^{\pi}$ and thus also of $X_{t}^{\pi}$ thereby minimizing the possibility of ruin. For this purpose, in the next subsection 5.1 we propose an algorithm of the type of policy improvement.

\subsection{Policy improvement}

Concerning the minimization of $l_{r}^{\pi}(t, k)$ at $r=R_{t}^{\pi}$ notice that decisions concerning the control actions $\phi=(b, \delta)$ have to be made only at the event times $T_{n}$. The minimization of $l_{r}^{\pi}(t, k)$ with respect to $\pi \in \mathcal{A}$ has thus to be performed only for pairs $(t, k)$ of the form $\left(T_{n}, K_{T_{n}}\right)$ thus leading to a policy $\pi$ with individual control actions $\phi_{T_{n}}=\left(b_{T_{n}}, \delta_{T_{n}}\right)$.

Our problem to determine an investment and insurance policy to minimize the bounds on the ruin probability may thus be solved by solving the following subproblems:

i) for a given policy $\bar{\pi} \in \mathcal{A}$ determine $l_{r}^{\bar{\pi}}(t, k)$ for pairs $(t, k)$ of the form $\left(T_{n}, K_{T_{n}}\right)$

ii) determine $R^{\bar{\pi}}\left(T_{n}, K_{T_{n}}\right)$ that is a solution with respect to $r$ of $l_{r}^{\bar{\pi}}\left(T_{n}, K_{T_{n}}\right)=$ 0 and put $R_{t}^{\bar{\pi}}=\inf _{t \leq T_{n} \leq T} \min \left[R^{\bar{\pi}}\left(T_{n}, 0\right), R^{\bar{\pi}}\left(T_{n}, 1\right)\right]$;

iii) improve the policy $\bar{\pi}$ by minimizing $l_{R^{\bar{\pi}}}^{\pi}\left(T_{n}, K_{T_{n}}\right)$ with respect to $\pi \in \mathcal{A}$.

This leads to a policy improvement-type approach, more precisely, one may proceed as follows:

a) start from a given policy $\pi^{0}$ (e.g. the one requiring minimal reinsurance and no investment in the financial market);

b) determine $R^{\pi^{0}}\left(T_{n}, K_{T_{n}}\right)$ corresponding to $\pi^{0}$ for the various $\left(T_{n}, K_{T_{n}}\right)$ as well as the ensuing $R^{\pi^{0}}$;

c) determine $\pi^{1} \in \mathcal{A}$ that minimizes $l_{R^{\pi^{0}}}^{\pi}\left(T_{n}, K_{T_{n}}\right)$ with respect to $\pi \in \mathcal{A}$ for the various $\left(T_{n}, K_{T_{n}}\right)$; repeat the procedure until a stopping criterion is met (notice that by the above procedure $R^{\pi^{n}}>R^{\pi^{n-1}}$ ). 
Notice that the policy determined by this procedure is automatically strongly admissible.

A practical way to implement steps b) and c) above is to discretize the time interval $[0, T]$ and then register an event only at the end of the interval in which it occurred (multiple events in a same subinterval may be recorded at the end as a single event for each of the two categories: claim, price change). The function $l_{r}^{\pi}(t, k)$ has then to be determined only for $t$ corresponding to an end point of the various subintervals for each of the two possible values of $k$. Analogously for $R^{\pi}$ as well as for the policy.

One crucial step in this procedure is to determine the function $l_{r}^{\pi}(t, k)$ corresponding to a given $\pi \in \mathcal{A}$ and for this purpose we can prove the following

Proposition 18 Under the standing assumptions we have that

$$
\begin{aligned}
l_{r}^{\pi}(t, k)= & (1-G(T-t)) e^{-r C(b)(T-t)}-1 \\
+ & \int_{0}^{T-t} e^{-r C(b) z} d G(z) . \\
\cdot & {\left[p_{k, 0} \int_{0}^{\infty} e^{r b y} d F(y)+p_{k, 1} \int_{\underline{w}}^{\bar{w}} e^{-r \delta\left(e^{w}-1\right)} d H(w)\right] }
\end{aligned}
$$

Proof: It is an immediate consequence of (4.1) and (4.2).

In the specific case described in Example 1, where $Z_{n}$ are i.i.d. having a negative exponential distribution with parameter $\lambda=\lambda_{0}+\lambda_{1}$ and where $p_{k, h}=\frac{\lambda_{h}}{\lambda},(h=0,1)$ independently of $k$, we have the following immediate corollary

Corollary 19 In the case of Example 1 one has

$$
\begin{aligned}
l_{r}^{\pi}(t, k) & =l_{r}^{\pi}(t) \\
& =e^{-(\lambda+r C(b))(T-t)}-1 \\
& +\frac{1}{\lambda+r C(b)}\left[1-e^{-(\lambda+r C(b))(T-t)}\right] . \\
& \cdot\left[\lambda_{0} \int_{0}^{\infty} e^{r b y} d F(y)+\lambda_{1} \int_{\underline{w}}^{\bar{w}} e^{-r \delta\left(e^{w}-1\right)} d H(w)\right]
\end{aligned}
$$

independently of the value of $k$. 
Remark 20 It can furthermore be shown, see Piscitello (2012), that in the case of Example 1 the value of $R^{\pi}(t, k)$ is not only independent of $k$ being a solution of $l_{r}^{\pi}(t)=0$, but it is furthermore independent also of $t$, so that the bound optimizing policy is stationary.

The expressions for $l_{r}^{\pi}(t, k)$ are thus easy to obtain provided the distributions of $Y_{1}$ and $W_{1}$ are such that the corresponding integrals are easy to compute. In any case they can be computed approximately by replacing the integrals with the corresponding Riemann-Stieltjes sums.

\section{Conclusions}

We have considered the problem of minimizing the ruin probability in an insurance model that allows to dynamically choose the level of reinsurance and investment in the financial market. It is a general innovative model that describes in a unifying way the timing of the events, that is the arrivals of claims and the changes of the prices in the market. It is based on a continuous-time Semi-Markov process model (SMP) and it is believed that this model represents reality more faithfully than, say, classical diffusion-based models. It leads also to some advantages when estimating the parameters in the model in the sense that it allows one to separate between the information coming from observing the frequency of the individual events and that of the duration between successive events. Our insurance model is also general enough to contain particular cases ranging from the classical risk process to models with reinsurance, investment in the financial market and dividends. It could possibly be extended to include also the recent general risk model with reinsurance in Eisenberg \& Schmidli (2010) where, to prevent a negative surplus, the insurer may inject additional capital. We recall also that our bounds were derived for the main purpose of allowing one to actually determine a reinsurance and investment policy that keeps the ruin probability at low levels.

We developed a specific methodology to obtain a policy that minimizes the exponential bound on the ruin probability in Theorem 15. It is based on a policy iteration procedure. Besides being of interest in itself, the bound minimizing policy that is obtained may also serve as a benchmark policy with respect to which other standard policies may then be evaluated.

Contrary to many asymptotic (in time and in the initial surplus) approaches (see e.g. Gaier et al. (2003), Paulsen (1998), Hult \& Lindskog 
(2010)) we obtain our results for a fixed, but arbitrary finite horizon and for any given positive initial surplus.

Acknowledgements: The authors gratefully acknowledge useful remarks by an anonymous referee, which allowed them to considerably reduce and streamline the original version of the paper.

\section{References}

Asmussen, S., Ruin Probabilities. World Scientific, River Edge, NJ. 2000.

Chen, S., Gerber, H. and Shiu, E., Discounted probabilities of ruin in the compound binomial model. Insurance: Mathematics and Economics, 2000, 26, 239-250.

Diasparra, M.A. and Romera, R., Bounds for the ruin probability of a discrete-time risk process. J. Appl. Probab, 2009, 46(1), 99-112.

Diasparra, M.A. and Romera, R., Inequalities for the ruin probability in a controlled discrete-time risk process. European Journal of Operational Research, 2010, 204(3), 496-504.

Edoli, E. and Runggaldier, W.J., On Optimal Investment in a Reinsurance Context with a Point Process Market Model. Insurance: Mathematics and Economics, 2010, 47, 315-326.

Eisenberg, J. and Schmidli, H., Minimising expected discounted capital injections by reinsurance in a classical risk model. Scand. Actuarial J., to appear, (first published March 2010).

Gaier, J.,Grandits, P. and Schachermayer, W., Asymptotic ruin probabilitites and optimal investment. Ann. Appl. Probab., 2003, 13, 1054-1076.

Grandell, J., Aspects of Risk Theory. Springer, New York. 1991.

Huang, T., Zhao, R. and Tang, W., Risk model with fuzzy random individual claim amount. European Journal of Operational Research, 2009, 192, 879-890. 
Hult, H. and Lindskog, F., Ruin probabilities under general investments and heavy-tailed claims. Finance and Stochastics, to appear, (published online on 21 August 2010).

Paulsen, J., Sharp conditions for certain ruin in a risk process with stochastic return on investment. Stochastic Processes and Their Applications, 1998, $75,135-148$.

Piscitello, C., Optimal reinsurance and investment in an insurance risk model (in Italian), Master's thesis, University of Padova, 2012.

Schäl, M., On Discrete-Time Dynamic Programming in Insurance: Exponential Utility and Minimizing the Ruin Probability. Scandinavian Actuarial Journal, 2004, 3, 189 - 210.

Schäl, M., Control of ruin probabilities by discrete-time investments. Math. Meth. Oper. Res., 2005, 62, $141-158$.

Schmidli, H., On minimizing the ruin probability by investment and reinsurance. Ann. Appl. Probab., 2002, 12, 890-907.

Schmidli, H., Stochastic Control in Insurance. Springer, London. 2008.

Xiong, S. and Yang, W.S., Ruin probability in the Cramér-Lundberg model with risky investments. Stochastic Processes and Their Applications, 2011, 121, 1125-1137.

Wang, R.,Yang, H. and Wang, H., On the distribution of surplus inmediately after ruin under interest force and subexponential claims. Insurance: Mathematics and Economics, 2004, 34, 703-714.

Willmot, G. and Lin, X., Lundberg Approximations for Compound Distributions with Insurance Applications Lectures Notes Statist. 156, Springer, New York, 2001. 